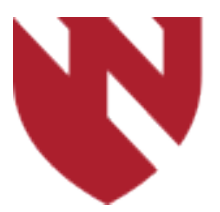

\title{
Nontuberculous Mycobacterium (NTM) Infection in Aquatic Workers with Purified Protein Derivative (PPD) and Gold QuantiFERON Tests.
}

\author{
Lavanya Ravipati \\ Clarkson Family Medicine \\ Sarah A. Howard \\ Clarkson Family Medicine
}

Tell us how you used this information in this short survey.

Follow this and additional works at: https://digitalcommons.unmc.edu/gmerj

Part of the Higher Education Commons, and the Infectious Disease Commons

\section{Recommended Citation}

Ravipati, L., , Howard, S. A. Nontuberculous Mycobacterium (NTM) Infection in Aquatic Workers with Purified Protein Derivative (PPD) and Gold QuantiFERON Tests.. Graduate Medical Education Research Journal. 2020 Dec 09; 2(2).

https://digitalcommons.unmc.edu/gmerj/vol2/iss2/8

This Case Report is brought to you for free and open access by DigitalCommons@UNMC. It has been accepted for inclusion in Graduate Medical Education Research Journal by an authorized editor of DigitalCommons@UNMC. For more information, please contact digitalcommons@unmc.edu. 


\title{
Nontuberculous Mycobacterium (NTM) Infection in Aquatic Workers with Purified Protein Derivative (PPD) and Gold QuantiFERON Tests.
}

\begin{abstract}
Background: Nontuberculous mycobacteria (NTM) infections are commonly caused by Mycobacterium marinum and fortuitum with $M$. marinum more likely to cause skin infections. Aquatic environments are a potential source of contact with M. marinum and fortuitum. Patients with NTM infections may present with positive tuberculin purified protein derivative (PPD) and/or gold QuantiFERON tests. However, PPD cannot differentiate between NTM infection and other tuberculous infections caused by different mycobacterial strains. We present a patient a marine biologist who was asymptomatic and presented for further evaluation as she has a h/o positive PPD and gold QuantiFERON test.

Methods: Patient informed consent was obtained for this case report.

Results: A 31 years old, healthy, female marine biologist employed in an aquatic environment presented to the clinic with a positive PPD and a positive gold QuantiFERON test. Her job exposes her to sea creatures that maybe infected with Mycobacterium marinum and fortuitum. She denied any history that was suggestive of tuberculous infection. A referral was made to specialist service for further evaluation at which time a repeat gold QuantiFERON test was performed and was noted to be positive. Following this work up, the patient preferred to follow a conservative treatment approach to monitor for any symptoms of active tuberculosis and seek follow up as needed.

Conclusion: Clinicians should not downplay the importance of aquatic occupations and hobbies in patients who exhibit positive PPD and gold QuantiFERON test. Healthcare providers should consider NTM infection in their differential diagnosis when examining patient populations exposed to aquarium water, saltwater, and freshwater environments. Preventative apparel like waterproof gloves can protect the exposed skin from infection. Clinicians can prevent NTM infections by increasing awareness and promoting prevention measures in at-risk populations, particularly since infection can be prevented from appropriate protective attire.
\end{abstract}

\section{Keywords}

Mycobacterium marinum, Mycobacterium fortuitum, PPD test, Gold QuantiFERON test, Aquatics, NTM (non-tuberculous mycobacterium), Granuloma.

\section{Creative Commons License}

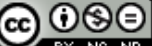

This work is licensed under a Creative Commons Attribution-Noncommercial-No Derivative Works 4.0 License. 


\section{Nontuberculous Mycobacterium (NTM) Infection in Aquatic Workers With Purified Protein Derivative (PPD) and Gold QuantiFERON Tests

\author{
Lavanya Ravipati ${ }^{1}$, Sarah A. Howard
}

${ }^{1}$ Clarkson Family Medicine, Omaha, NE

\begin{abstract}
Nontuberculous mycobacteria (NTM) infections are commonly caused by Mycobacterium marinum and fortuitum with $M$. marinum more likely to cause skin infections. Aquatic environments are a potential source of contact with $M$. marinum and fortuitum. Patients with NTM infections may present with positive tuberculin purified protein derivative (PPD) and/or gold QuantiFERON tests. However, PPD cannot differentiate between NTM infection and other tuberculous infections caused by different mycobacterial strains. We present a patient (a marine biologist) who was asymptomatic and presented for further evaluation as she has a history of positive PPD and gold QuantiFERON test. She denied any history that was suggestive of tuberculous infection. A referral was made to specialist service for further evaluation, at which time a repeat gold QuantiFERON test was performed and was noted to be positive. Following this work up, the patient preferred to follow a conservative treatment approach to monitor for any symptoms of active tuberculosis and seek follow up as needed. Clinicians should not downplay the importance of aquatic occupations and hobbies in patients who exhibit positive PPD and gold QuantiFERON test. Healthcare providers should consider NTM infection in their differential diagnosis when examining patient populations exposed to aquarium water, saltwater, and freshwater environments. Preventative apparel such as waterproof gloves can protect the exposed skin from infection. Clinicians can prevent NTM infections by increasing awareness and promoting prevention measures in at-risk populations, particularly since infection can be prevented from appropriate protective attire.
\end{abstract}

\section{Introduction}

Nontuberculous mycobacteria (NTM) infections are commonly caused by Mycobacterium marinum and fortuitum with M. marinum more likely to cause skin infections. Aquatic environments, with exposure to aquatic animals, are a potential source of contact with $M$. marinum and fortuitum. As a result, employees working in such environments are at a higher risk of infection from these organisms. These pathogens can cause contact zoonoses, mainly affecting the skin, while having a chronic indolent and benign course. ${ }^{1}$ Patients with NTM infections may present with positive tuberculin purified protein derivative (PPD) and/or gold QuantiFERON tests. However, PPD cannot differentiate between NTM infection and other tuberculous infections caused by different mycobacterial strains. Similarly, PPD cannot differentiate NTM infection and Bacillus Calmette-Guerin vaccine (BCG) reactivity, as PPD contains both Mycobacterium tuberculosis (MTB) and NTM antigens. ${ }^{1}$ However, recombinant early secreted antigen target 6 (rESAT6) is an antigen used in gold QuantiFERON tests and can elicit a positive test when humans are exposed to MTB or any other mycobacterium such as M. marinum that contain the gene for rESAT6. ${ }^{2}$ Thus, it is vital for clinicians to be aware of specific at-risk populations and assist with prevention measures and treatment strategies. ${ }^{1}$

\section{Case Presentation}

A 31-year-old female marine biologist presented to the clinic with a positive PPD (May 2017). She also had a positive gold QuantiFERON test (Dec 2017). She is employed in an aquatic environment which necessitates contact with sea creatures that maybe infected with or have died from Mycobacterium marinum and fortuitum. She treats the habitat, does necropsies, and has found that they have granulomas consistent with NTM disease. At the start of the employment, the patient tested negative for PPD and then became reactive later, similar to co-workers, some of whom have tested positive for tuberculosis. She did not give any history of fever, chills, night sweats, hemoptysis, weight loss, cough, or any other active symptoms of tuberculosis. She denied any history of any other TB exposure.

On physical examination she did not show signs of skin lesions anywhere on the body. A chest x-ray was obtained in clinic and was normal. She was asymptomatic and seemed to be in good health. A referral was made to specialist service for further evaluation, at which time a repeat gold QuantiFERON test was performed and was noted to be positive. Following this work up, the patient preferred to follow a conservative treatment approach to monitor for any symptoms of active tuberculosis and seek follow up as needed.
Consent to use this case for educational purposes was obtained from the patient.

\section{Discussion}

It is likely that the patient's clinical picture could be due to NTM infection due to occupational exposure to sea animals that harbor M. marinum and fortuitum. $M$. marinum is more likely to cause skin infections compared to $M$. fortuitum because it exhibits preferential growth at temperatures less than $37 \mathrm{C}$ associated with cooler parts of the body like the skin. ${ }^{3,4}$ NTM infection causing $M$. marinum was first isolated in 1926 from salt water fish carcasses at the Philadelphia Aquarium. ${ }^{5}$ The bacterial pathogen was later isolated in swimmer's skin lesions and recognized as a human pathogen in 1951. ${ }^{5}$ After swimming pool chlorination became standard, most reported cases of M. marinum infection were in fisherman and aquarium owners who exhibited skin lesions/ infection. Thus, the term "Fish tank granuloma" was coined. ${ }^{6}$ Most M. marinum NTM infections are acquired through wounds that are in contact with aquarium water, contaminated salt water, or fresh water. ${ }^{3,6} \mathrm{~A}$ staggering 50 to $84 \%$ of cases are aquarium owners. $^{3}$

\section{Although a positive PPD and gold QuantiFERON test can be indicative of NTM infection, many patients may not exhibit any symptoms. Typical presentation of NTM infection can include a tender erythematous nodule, pustule at the site of inoculation, and ulcerations. ${ }^{1,6}$ More severe cases with deeper infection can be associated with tenosynovitis, septic arthritis, and osteomyelitis. ${ }^{6,7}$ In immunocompetent individuals, infection usually remains localized and single lesions can remit spontaneously with time. Whereas in immunocompromised individuals, dissemination of the pathogen can still occur causing more invasive skin infections and requiring immediate treatment. ${ }^{3,6,8}$}

It is vital for health care providers to take note of their patient's occupational history and other personal activities to isolate any sources of exposure to M. marinum. A 10-year study in Bangkok examining M. marinum cases found that $66.7 \%$ of infected patients had occupations or hobbies putting them in direct contact with fish and water. ${ }^{5}$ Further, a positive PPD and gold QuantiFERON test 
can be easily misdiagnosed for the more common M. tuberculosis-related infection. This can lead to additional testing, such as chest $\mathrm{X}$-rays, and inappropriate treatment that can cause an increased burden on the U.S. healthcare system. Through genetic analysis, $M$. marinum is found to be closely related to M. tuberculosis, so both pathogens can elicit sufficient tuberculin skin test reactivity yielding a positive PPD as well as the gold QuantiFERON test. ${ }^{4}$

When there is any suspicion of $M$. marinum infection, clinicians should perform a mandatory biopsy followed by histopathology and tissue culture to confirm the diagnosis. ${ }^{1}$ The gold standard for the diagnosis of mycobacterial infection is a positive culture from the tissue biopsy. ${ }^{3}$ Culture in an appropriate medium and polymerase chain reaction- restriction enzyme analysis (PRA)-hsp65 analysis can help confirm the diagnosis. ${ }^{1}$ While there is no standard treatment recommendation, $M$. marinum is generally sensitive to an array of antibiotics such as clarithromycin, sulfamethoxazole/ trimethoprim and minocycline. ${ }^{1,6}$ Even if the organism is deemed susceptible to an antibiotic, mono therapy should be avoided because of the unreliability of susceptibility testing for this organism and the possibility of emerging resistance. ${ }^{4}$

Evaluation for tuberculosis must always be performed in those with positive tuberculin test and treatment provided based on the current guidelines. ${ }^{4}$ As noted in the Dartmouth- Hitchcock Medical Center, New Hampshire study, all patients with NTM tested had tuberculin reactions of $>10-15$ $\mathrm{mm} .{ }^{4}$ Hence infection with these organisms is associated with sufficient tuberculin skin test reactivity to trigger consideration of treatment for latent TB. ${ }^{4}$ However, positive tuberculin reactions in patients with $M$. Marinum infection can be attributed to this infection and should not always be considered an indication for treatment of latent tuberculosis. ${ }^{4}$

\section{Conclusion}

Clinicians should not downplay the importance of aquatic occupations and hobbies in patients who exhibit positive PPD and gold QuantiFERON test. Healthcare providers should consider NTM infection in their differential diagnosis when examining patient populations exposed to aquarium water, saltwater, and freshwater environments. ${ }^{3,4,6}$ Since $M$. marinum infection is not common, dermatologic practices and other providers have low awareness of the condition. ${ }^{3}$ Untreated patients can progress to serious complications ranging from septic arthritis and osteomyelitis to disseminated disease in immunocompromised patients. ${ }^{6}$ Preventative apparel like waterproof gloves can protect the exposed skin from infection. ${ }^{1,4}$ Clinicians can prevent NTM infections by increasing awareness and promoting prevention measures in at-risk populations, particularly since infection can be prevented from appropriate protective attire.

https://doi.org/10.32873/unmc.dc.gmerj.2.2.010

\section{References}

1 Christiane Salgade Sette, Patrick Alexander Wachholz, Paula Yoshiko Masuda, et al. Mycobacterium marinum infection: a case report. Journal of Venomous Animals and Toxins including Tropical diseases. (2015) 21:7 DOI 10.1186/s40409-015-0008-9

$2 \mathrm{X} \mathrm{Wu}, \mathrm{L}$ Zhang, J Zhang, et al. Recombinant early secreted antigen target 6 protein as a skin test antigen for the specific detection of mycobacterium tuberculosis infection. Clinical \& Experimental Immunology 2008 Apr;152(1): 81-87. doi: 10.1111/ j.1365-2249.2008. 03605.x

3 M. Dolenc-Voljč and M. Žolnir-Dovč. Delayed diagnosis of Mycobacterium marinum infection: A case report and review of the Literature. Acta Dermatoven APA Vol 19 2010, No 2.

4 Felicia. M.T. Lewis, Bryan J Marsh, and C. Fordham Von Reyn. Fish Tank Exposure and Cutaneous Infections Due to Mycobacterium Marinum: Tuberculin Skin testing, Treatment and Prevention. Clinical Infectious Diseases 2003; 37:390-7.

5 Por Ang MD, Niramol Rattana-Apiromyakij MD, Chee-Leok Goh MD. Retrospective study of Mycobacterium Marinum skin Infections - Ang - 2000 - International Journal of Dermatology. https://onlinelibrary.willey.com/doi/ full/10.1046/j.1365-4362.2000. 00916.x

6 CPT Ryan Johnson, CPT Yang Xia, CPT Sunghun Cho, et al. Mycobacterium marinum Infection: A Case Report and review of the Literature. Continuing Medical Education Albert Einstein College of Medicine. 2006 CUTIS 2007;79:33-36.

7 Sonia Ferdinand, Theresa M Meloche, Tony L Brown. Mycobacterium Marinum: a case report. Journal of Bacteriology and Mycology: Open Access. 2016;2(4): 98-100.

8 B. Petrini. Mycobacterium Marinum: ubiquitous agent of waterborne granulomatous skin infections. European Journal of Clinical Microbiology and Infectious Diseases. October 2006, Volume 25, Issue 10 , pp 609-613/Cite as. 